\title{
GRACILE syndrome
}

INSERM

\section{Source}

INSERM. (1999). Orphanet: an online rare disease and orphan drug data base. GRACILE syndrome. ORPHA:53693

GRACILE syndrome is an inherited lethal mitochondrial disorder characterized by fetal growth restriction (GR), aminoaciduria (A), cholestasis (C), iron overload (I), lactacidosis $(L)$, and early death (E). 